\title{
EDITORIAL
}

\section{Molecular cytogenetics: karyotype evolution, phylogenomics and future prospects}

Heredity (2012) 108, 1-3; doi:10.1038/hdy.2011.117

Cytogenetics has matured into a multidisciplinary science that draws heavily on theoretical and technical advances associated with developments in molecular biology, flow-cytometery, bioinformatics and phylogenetics. As a result, it now provides crucial analytical approaches for countless questions in evolutionary biology. Furthermore, its increasing reliance on sequence data to confirm and refine syntenies, identify breakpoints, neocentromeres and other chromosomal features has irrevocably cemented the association between molecular cytogenetics, karyotypic change and genomics-aspects that form the focus of this Special Issue.

The historic journey of the field from the use of sectioned or squash preparations of tissues in situ through the discovery of a hypotonic pretreatment for spreading chromosomes, a key development that permitted the accurate determination of chromosome numbers and morphology, to the discovery of differential banding patterns, has been well documented (Hsu, 1979). The idea that evolutionary relationships may be inferred from structural as well as banding details ('bar codes of homologous segments among species', O'Brien et al., 2006) was energized by the advent of molecular cytogenetics. Specifically, the discovery that fluorescently labelled DNA probes (produced from flow-sorted chromosomes, microdissected chromosomes and cloned DNA libraries) could be hybridized to chromosomes fixed on microscope slides was pivotal to the development of the field. For the first time, the unequivocal identification of regions of orthology was made possible by direct observation based on DNA sequence location, rather than on morphology and banding patterns (see Ferguson-Smith and Trifonov, 2007 and references therein). This led to a shift from the frequent and somewhat anachronistic use of comparative cytogenetics as a largely descriptive science in systematic studies, to investigations of the mode (including epigenetic modification), tempo and degree to which chromosomal change underpins the diversification of lineages, from population analysis to deep phylogenetic divisions. This new comparative approach, together with the increasing availability of genome sequences and improved methods of annotation, has provided novel insights into karyotype evolution and a more coherent view of the evolutionary history of different genomes.

This special issue includes primary articles and reviews (several of which contain significant new data) that explore one or more of the themes noted above. Romanenko et al. (2012), for example, review the contribution that cross-species fluorescence in situ hybridization (zooFISH) has made to our understanding of chromosome evolution in Rodentia. Their data permit a refinement of several presumptive ancestral karyotypes whose constituent species are often characterized by highly rearranged genomes. Whole chromosome painting probes (that is, those amplified from single chromosome templates), however, detect only a subset of chromosomal rearrangements thus missing intrachromosomal changes such as inversions and duplications as well as small translocated segments that are beyond the resolution of FISH (5-10 Mb; Schrock et al., 1996). Refinements, designed to improve the resolution of conserved syntenic blocks identified using wholechromosome paints from conserved genomes, include the use of ordered subchromosomal markers such as BACs, and/or of highly fragmented genomes as painting probes. Nie et al. (2012) demonstrate this in their study of chromosomal evolution in Carnivora. Using chromosome-specific paints derived from the domestic dog (that has a high chromosome number, $2 n=78$, and one of the most rearranged karyotypes in mammals) they present comparative homology maps from the species representative of six different families. Together with data from human-carnivore and carnivore-specific painting studies, their high-resolution cytogenetic analysis reveals inversion differences among taxa that would not be detected using probes from syntenyconserved genomes such as those of the human and the cat.

One of the most important outcomes of zoo-FISH experiments is the detection of conserved chromosomes, chromosomal segments and segmental associations that together correspond to intact ancestral chromosomes. A limitation of this approach is that increasing evolutionary distance between the species reduces resolution, hindering attempts to paint across the eutherian/metatherian boundary as well as to more distant lineages. Nonetheless, conserved syntenies detected by FISH could be retrieved by in silico scans of the genome assemblies of phylogenetically distantly related vertebrates leading to hypothesized ancestral genomic configurations for groupings in placental mammals, as well as amniotes, tetrapods and even vertebrates. Using this approach, Ruiz-Herrera et al. (2012) extend the in silico identification of conserved segmental associations at deeper nodes of the vertebrate tree through the inclusion of data from a recently sequenced Xenopus genome. Although the incomplete nature of the assemblies available for both platypus and the frog impact the ability to determine whether entire segments have been retained, of the nine ancient syntenic segmental associations identified in conserved karyotypes of extant placentals (thought likely to reflect the structure of ancestral chromosomes for eutherians), six predate the divergence of tetrapods.

Yet another intriguing outcome of whole-genome analysis of this kind is the finding that regions rich in segmental duplications and repetitive elements of various types (including retroposons) show reuse as regions of chromosomal breakage (that is, evolutionary breakpoint regions). Although this phenomenon (breakpoint reuse) has been well documented in mammals (for example, Murphy et al., 2005), similar findings appear to hold also for the Aves. Although the overwhelming pattern of chromosomal evolution in the birds is one of constrained change, using whole-chromosome sequences of three 
avian species (zebra finch, chicken and turkey) Skinner and Griffin (2012) unmask a surprisingly high number of intrachromosomal rearrangements (inversions) that distinguish these species. They report that a third of breakpoint regions in chicken macrochromosomes 1-10 reoccur in the zebra finch and turkey orthologs, and that $\sim 10 \%$ of the evolutionary breakpoint regions reoccur in different lineages.

No treatment of chromosome evolution is complete without reference to the mechanisms of change that are responsible for the myriad of karyotypes that characterize biological lineages. Although there is certainly strong evidence to support the idea that chromosomal breakpoints are nonrandom, and there is a causal relationship between segmental duplication, repetitive elements, fragile sites and evolutionary breakpoint reuse at the junction of conserved syntenies, a link between these 'hotspots' of genome instability and non-coding RNAs (ncRNAs) also suggests an epigenetic component to chromosome rearrangement. In their paper, Brown et al. (2012) focus on the role of ncRNAs as mediators of epigenetic regulation and their involvement in structural change. They provide tantalizing insights into the dynamic processes that govern chromosomal condensation into euchromatin and heterochromatin, and suggest chromatin modification is not only reliant on DNA sequence composition, but also that it requires complex epigenetic modifications that are often dependent on ncRNAs. They propose that ncRNAs cause a delay in replication timing resulting in chromosomal instability, and can contribute to evolutionary novelty through neocentromere formation, translocations and other modifiers of chromosomal morphology.

Long ncRNAs are also important regulators of, among others, genomic imprinting in mammals, perhaps best exemplified by $\mathrm{X}$ chromosome inactivation, a phenomenon that evolved to correct dosage imbalance of genes on the sex chromosomes. Unlike autosomes, where there is strict registry of gene loci along the length of homologues, the presence of differentiated sex chromosomes that were once homologous (XY in mammals, and ZW in birds, snakes and some lizards and fish) reflects the progressive degradation and diminution of the sex-specific, non-recombining Y (or W) after its acquisition of a sex-determining function. Livernois et al. (2012) track the evolution of sex chromosomes and dosage compensation in amniotes. They draw on both molecular cytogenetics and comparative genomics to show that the $\mathrm{X}$ in Placentalia is well conserved in size and gene order across taxa, the marsupial $X$ shares partial homology with it, and that the corresponding segments are all autosomal in monotremes. Moreover, although the sex chromosomes of birds are superficially similar to placentals, comparative gene mapping has shown that they are genetically non-homologous, a situation mirrored by the lack of homology between bird and snake $\mathrm{Z}$ chromosomes.

Centromere repositioning is a recent addition to the suite of mechanistic processes that shape karyotype evolution. Although difficult to unambiguously define with conventional cytogenetics (that is, banding in conjunction with whole-chromosome paints), and often hard to discern from sequence data, BAC-FISH with precisely mapped markers has proved an effective means of tracking centromere movement. Rocchi et al. (2012) provide a timely review of 'evolutionary new centromere (ENC) occurrence', which suggests that in some species such as primates and equids, centromere repositioning occurs as a result of the emergence of a new centromere rather than by transposition of an existing one. Population data that reveal the early stages of the evolution of an ENC (that is, in progress) are virtually nonexistent, and the authors' observations that reseeding of a centromere is responsible for the polymorphic state of chromosome 9 in orangutan is unprecedented. Its presence as a polymorphism in the Bornean and Sumatran species suggests that it predated their insular geographic isolation and survived a speciation event. Britton-Davidian et al. (2012) report an equally intriguing population-level analysis on the most ubiquitous of small mammals, the polytypic house mouse, Mus musculus. The authors' analysed the number and location of nucleolar organizing regions (NORs) in specimens drawn from localities in Asia to Western Europe and show a surprisingly high rate of polymorphism for the presence/absence of rDNA clusters on a large number of chromosomes. They argue that the most likely explanation for the observed patterns of NOR occurrence among the five house mouse subspecies favours lineage sorting of ancestral NOR polymorphisms. In this respect, NORs function as neutral genetic markers and, consistent with molecular phylogenetic studies, suggest a pattern that tracks stochastic processes (drift, founder effects, gene flow) that occurred in the radiation of the different sublineages.

The final paper in this special issue (Le Scouarnec and Gribble, 2012) illustrates how advances in array-based assays and next generation sequencing technologies facilitate our ability to identify and characterize pathological chromosomal rearrangements and genomic structural variation with precision. Although focused on human clinical genetic problems, these technologies have potential to be expanded to a diversity of research projects involving non-human genomes. For example, the current low-resolution analysis of evolutionary breakpoint regions by conventional zoo-FISH will be significantly improved through cross-species array painting. This will permit an appraisal of the composition of breakpoints (repetitive sequence and gene content, epigenetic modification, centromere seeding) and thus a more robust determination of their reuse during karyotypic evolution.

In conclusion, the papers in this Special Issue demonstrate the current trends and developments in the discipline. Yet, serious challenges lie ahead. Although population surveys will continue to inform our knowledge of the behaviour of nascent rearrangements and thus shed light on chromosomal speciation, our understanding of what role, if any, chromosomal rearrangements have had in the adaptive radiation of vertebrates is far from complete. In spite of new technological advances, and the unprecedented availability of genome sequences, this may prove the most testing challenge yet.

\section{CONFLICT OF INTEREST}

The authors declare no conflict of interest.

TJ Robinson' ${ }^{1}$ and F Yang ${ }^{2}$
${ }_{1}^{1}$ Evolutionary Genomics Group, Department of Botany and Zoology,
University of Stellenbosch, Matieland, South Africa and
${ }^{2}$ The Wellcome Trust Sanger Institute,
Wellcome Trust Genome Campus, Cambridge, UK

E-mail: tjr@sun.ac.za

Britton-Davidian J, Cazaux B, Catalan J (2012). Chromosomal dynamics of nucleolar organizer regions (NORs) in the house mouse: micro-evolutionary insights. Heredity 108: 68-74

Brown JD, Mitchell SE, O'Neill RJ (2012). Making a long story short: noncoding RNAs and chromosome change. Heredity 108: 42-49.

Ferguson-Smith MA, Trifonov V (2007). Mammalian karyotype evolution. Nat Rev Genet 8 : 950-962.

Hsu TC (1979). Human and Mammalian Cytogenetics_an Historical Perspective. Springer Verlag: New York, NY.

Le Scouarnec S, Gribble SM (2012). Characterising chromosome rearrangements: recent technical advances in molecular cytogenetics. Heredity 108: 75-85.

Livernois AM, Graves JAM, Waters PD (2012). The origin and evolution of vertebrate sex chromosomes and dosage compensation. Heredity 108: 50-58. 
Murphy WJ, Larkin DM, Everts-van der Wind A, Bourque G, Tesler G, Auvil L et al. (2005). Dynamics of mammalian chromosome evolution inferred from multispecies comparative maps. Science 309: 613-617.

Nie W, Wang J, Su W, Wang D, Tanomtong A, Perelman PL et al. (2012). Chromosomal rearrangements and karyotype evolution in carnivores revealed by chromosome painting. Heredity 108: 17-27.

O'Brien SJ, Menninger JC, Nash WG (2006). An Atlas of Mammalian Chromosomes. John Wiley \& Sons, Inc.: Hoboken, NJ.

Rocchi M, Archidiacono N, Schempp W, Capozzi O, Stanyon R (2012). Centromere repositioning in mammals. Heredity 108: 59-67.
Romanenko SA, Perelman PL, Trifonov VA, Graphodatsky AS (2012). Chromosomal evolution in Rodentia. Heredity 108: 4-16.

Ruiz-Herrera A, Farré M, Robinson TJ (2012). Molecular cytogenetic and genomic insights into chromosomal evolution. Heredity 108: 28-36.

Schröck E, du Manoir S, Veldman T, Schoell B, Wienberg J, Ferguson-Smith MA et al. (1996). Multicolor spectral karyotyping of human chromosomes. Science 273: 494-497.

Skinner BM, Griffin DK (2012). Intrachromosomal rearrangements in avian genome evolution: evidence for regions prone to breakpoints. Heredity 108: 37-41. 